\title{
"Ser alguien": motivaciones de estudiantes con Necesidades Especiales en Enseñanza Secundaria técnico profesional
}

\author{
Marcela Salazar Ramos ${ }^{1}$ \\ ORCID: 0000-0003-4732-3416 \\ Pedro Quiroga Menéndez ${ }^{1}$ \\ ORCID: 0000-0003-1887-7743 \\ Ximena Risco Neira ${ }^{1}$ \\ ORCID: 0000-0001-8640-2737
}

\section{Resumen}

El siguiente artículo aborda los resultados de un estudio cualitativo efectuado en colegios técnico-profesionales de Santiago de Chile, cuyo objetivo fue indagar en las motivaciones y expectativas de estudiantes con Necesidades Educativas Especiales (NEE) para estudiar una carrera técnica. La enseñanza media técnico profesional ha sido tradicionalmente connotada como un camino más directo al empleo y en la que subyace el supuesto de que es casi la única o principal posibilidad educativa para estratos populares. La muestra se compuso de 37 estudiantes de ambos géneros, de tercer y cuarto año, socioeconómicamente vulnerables, que presentaban alguna NEE y que eran atendidos por los programas de integración escolar (PIE) de sus respectivos colegios. El análisis de datos operó a través del Método de Comparación Constante, que codificó y refinó categorías hasta acceder a comprensiones teóricas emergentes. Los resultados muestran que los estudiantes perciben sus entornos escolares como integradores y protectores ante sus situaciones de discapacidad y necesidades especiales, valoran la formación técnica como una etapa que los puede proyectar eventualmente hacia la Educación Superior y sus expectativas se asocian a oportunidades de empleo y/o de estudios superiores. La evidencia sugiere que estamos ante algunos signos de cambio y adaptación del paradigma educativo, ya que gradualmente se transita desde esquemas compensatorios e integrativos hacia un diseño más inclusivo de la diversidad, lo que desde un enfoque de derechos debería impulsar el fortalecimiento de estrategias que superen la naturalizada oposición de normalidad/ anormalidad que aún se aprecia en nuestros sistemas escolares.

\section{Palabras clave}

Integración educativa - Necesidades Educativas Especiales (NEE) - Motivaciones y expectativas estudiantiles - Enseñanza media técnico profesional - Inclusión.

1- Universidad Tecnológica de Chile Inacap, Santiago, Chile. Contactos: marcela.salazar@inacapmail.cl; pquiroga@inacap.cl; xrisco@inacap.cl (7) DOl: http://dx.doi.org/10.1590/S1678-4634201945192699 


\section{"To be someone": motivations of students with Special Needs in Technicaland Vocational Secondary Education}

\section{Abstract}

This article shows the results of a qualitative research in technical and vocational schools in Santiago of Chile, whose objective was to inquire about SEN (Special Education Needs) students' motivations and expectations seeking a technical career. High school education, specialized in technical and professional teaching, has traditionally been seen as a more direct path to employment with the underlying assumption that it is almost the only or major educational opportunity for the poor. The sample is composed of 37 students of both sexes, $3 r d$ and 4 th graders in high school, socioeconomically vulnerable, holding any type of SEN, who were served by the SIP (School Integration Program) of their respective high schools. Data analysis was conducted through the Constant Comparison Method, which codified and refined categories until it was possible to access emerging theoretical frameworks. The results show that students perceive their school environment as integrative and protective upon their disability and special needs, they value the technical formation as a stage that may push them forward towards the university, and their expectations are associated with job opportunities and higher education. The evidence suggests that there are a few signs of change and adaptation of the educational paradigm, since it gradually shifts from countervailing and integrative schemes to a more inclusive design of diversity which, from a rights' approach should boost and strength strategies that overcome the naturalized opposition of normality/abnormality that can still be observed in our schools.

\section{Keywords}

Educational integration - Special Educational Needs (SEN) - Student motivations and expectations - Technical and vocational secondary education - Inclusion.

\section{Introducción}

En la actualidad, existe un relativo consenso acerca de la importancia de entender las condiciones que caracterizan la formación secundaria técnico profesional de jóvenes con necesidades educativas especiales (en adelante NEE). Una mirada al contexto nacional muestra que, en la actualidad, la educación chilena está transitando un nuevo período de reforma en todos sus niveles y modalidades. Las tensiones de este proceso se dejan sentir en distintos frentes, entre los que está la manera de cómo resolver la inclusión de estos estudiantes al sistema educativo $\mathrm{y}$, de este modo, cumplir con la promesa democrática de accesibilidad y dignidad para toda persona, superando así la débil integración que predomina actualmente en el sistema educativo y social.

Para captar el estado de la integración/inclusión escolar y social, despejando los factores que la rodean, es esencial atender primero a las definiciones de base con 
"Ser alguien": motivaciones de estudiantes con Necesidades Especiales en Enseñanza secundaria técnico...

las que el propio sistema social opera en cuanto a lo que involucra ser un estudiante con NEE en él. Para el sistema escolar, según la Ley 20.201, "Un alumno que presenta necesidades educativas especiales, es aquel que precisa de ayudas y recursos adicionales, ya sean humanos, materiales o pedagógicos, para conducir su proceso de desarrollo y aprendizaje, y contribuir al logro de los fines de la educación” (CHILE, 2009, p. 9) y debe estar obligatoriamente diagnosticado por un profesional médico, para que se reconozca dicha condición, de lo que subrepticiamente se desprende su noción de anormalidad o patología. Conforme al mismo decreto, las NEE se clasifican en dos tipos, permanentes y transitorias, diferenciadas según la complejidad y características que ellas tengan para considerar apoyos y ajustes en las condiciones de infraestructura y estrategias educativas hacia los estudiantes que las presentan. Ahora bien, cuando esta definición de NEE se contrasta con el concepto disciplinar e histórico de Educación especial, se observa un matiz diferenciador interesante, ya que en él, las NEE abarcan a "[...] cualquier niño/a o joven que requiera de algún tipo de adaptaciones específicas en su proceso educativo para lograr niveles de aprendizaje adecuados a sus potencialidades” (GÓNGORA, 2009, p. 21) por lo que la necesidad educativa en sí misma no debería ser sinónimo de enfermedad o trastorno ni tampoco debería implicar un diagnóstico profesional que lo clasifique y que condicione su acceso a derechos, así como tampoco debería ponerse énfasis en la condición supuesta de anormalidad o patología del estudiante sino en sus potencialidades, pero sobre todo en la capacidad del contexto escolar para adecuarse a ellos, es decir, existe una perspectiva inclusiva respecto a cómo entender y atender dichas condiciones, toda vez que la situación del estudiante es resultado de la interacción con el entorno y no solo de sí mismo. En este punto, es necesario señalar que en la actualidad conviven una diversidad de enfoques y miradas sobre inclusión educativa y que el concepto mismo se encuentra en tránsito desde definiciones disciplinarias y políticas; no obstante, un buen punto de partida lo ofrece la definición de la UNESCO, cuando señala que

Se puede concebir el concepto más amplio de educación inclusiva como un principio rector general para reforzar la educación para el desarrollo sostenible, el aprendizaje a lo largo de toda la vida para todos y un acceso a las oportunidades de aprendizaje en condiciones de igualdad para todos los niveles de la sociedad. (UNESCO, 2008, p. 3).

Las comprensiones de esta idea de inclusión oscilan desde enfoques de tipo macro, como los análisis filosóficos o socio antropológicos, hasta enfoques de tipo micro, a nivel de lo pedagógico y psicopedagógico, aun cuando puede considerarse que ambos niveles son complementarios en la medida en que atienden a factores holísticos y pertenecen al fenómeno de exclusión y al modo en que sus interrelaciones inciden en la escuela desde fuera de ella (tales como la historia y cultura de una región, su economía, políticas educativas, entre otros elementos más estructurales) o desde su interior (como por ejemplo las concepciones y prácticas pedagógicas) en las que se pretende incidir para lograr una mayor equidad educativa (ECHEITA, 2013). Desde la perspectiva de algunas organizaciones internacionales, el elemento de fondo que se debería resaltar es la equidad educativa, entendiéndola como "[...] una cuestión de justicia que implica garantizar que 
las circunstancias personales y sociales (género, nivel socioeconómico, procedencia, capacidad, etc.) no deben ser un obstáculo para el logro educativo” (OCDE, 2007, p. 11).

Asentadas estas primeras precisiones, cabe decir que el debate sobre inclusión en educación es de reciente atención en Chile si se compara con otras latitudes en que ya es de antigua data. En efecto, la revisión de algunos estudios estadounidenses muestra que ya en los años '80 investigadores como Mori analizaban la problemática de la formación profesional de los jóvenes con discapacidades de aprendizaje (learning disabilities) mostrando que se les había prestado poca atención a las posibilidades de educación superior de aquellos y que era clave profundizar sobre este tema en miras a la obtención de resultados de empleabilidad y de vida independiente. Mori también resaltaba que los sistemas que trabajaban con jóvenes de educación media estaban escasamente involucrados en la educación orientada a la enseñanza superior y a las actividades de preparación ocupacional. El autor resaltaba que las condiciones de integración de estos estudiantes a clases vocacionales o técnicas, a menudo los exponía a maquinarias, procedimientos y técnicas con poco apoyo y a estándares de desempeño no realistas, por lo que se debían hacer cambios para que la educación especial y la vocacional colaboraran para responder a los problemas específicos de la preparación para una carrera de estudiantes con necesidades especiales (MORI, 1980).

Por su parte, en 2005 Luftig y Muthert se interesaron por la perspectiva de la transición desde el colegio al trabajo y adultez, investigando la formación y empleabilidad de personas con discapacidades de aprendizaje. Estos autores concluyeron que los adultos con discapacidades de aprendizaje específicas o leve retraso mental que se habian graduado o egresado de una escuela secundaria inclusiva que enfatizaba el entrenamiento vocacional tecnológico y las habilidades de vida independiente, tenían resultados de empleabilidad más altos que el promedio nacional y unos rangos de sueldo mejores que los indicadores esperados para una vida independiente; en comparación, hasta el 75\% de personas que presentaban discapacidades leves, graduados en escuelas secundarias comunes o matriculados en programas académicos primarios o en programas con entrenamiento en educación vocacional a medio tiempo, se encontraban desempleados o subempleados hasta tres años después de egresar.

Más recientemente y también en EEUU, en el contexto del movimiento nacional hacia estándares más altos de la educación, el segundo Estudio de Transición Longitudinal (National Longitudinal Transition - NLTS2) examinó las carreras e inscripción a cursos en educación técnica de estudiantes de enseñanza secundaria con discapacidades de aprendizaje, para determinar si los estudiantes egresan de la educación secundaria listos para la universidad. Los análisis descriptivos documentan que aunque en la enseñanza secundaria existe una extensión general en la inscripción de cursos técnicos de parte de estos estudiantes y que se observa su compromiso en programas ocupacionales específicos, la evidencia disponible no muestra beneficios en el hecho de elegir especialidades técnicas en general, pero sí muestra que habría un efecto positivo significativo al participar de un conjunto de ocupaciones técnicas específicas en los primeros dos años postsecundaria, lo cual situaría a "[...] la juventud con discapacidades de aprendizaje en una trayectoria positiva en miras a obtener empleos competitivos de tiempo completo, 
"Ser alguien": motivaciones de estudiantes con Necesidades Especiales en Enseñanza secundaria técnico...

con los correspondientes salarios más altos, y la probabilidad de beneficios pagados por sus empleadores.» (WAGNER; NEWMAN; JAVITZ, 2015, p. 8). Todos estos antecedentes sugieren entonces que una formación secundaria técnico profesional es crucial para impactar en las condiciones laborales y sociales de estos jóvenes, ofreciendo interesantes claves acerca de cómo debería ser asumida esa formación, ya sea como una meta en sí misma o como una antesala para otras etapas de educación superior.

Volcando la mirada a la realidad chilena, la enseñanza media técnico profesional (EMTP) aparece representada en el imaginario colectivo, de manera general, como una educación para el empleo, que habilita para lo productivo. Distintas referencias nacionales e internacionales recientes -Larrañaga, Cabezas y Dusaillant (2013) y Arias y otros autores (2015), entre otros- demuestran explícitamente que la EMTP en Chile es parte relevante de la oferta formativa del sistema educativo, representando el $44 \%$ de la cobertura de matrícula de $3^{\circ}$ y $4^{\circ}$ año de Enseñanza Media y que se caracteriza por acoger a un importante segmento de estudiantes procedentes principalmente de sectores de alta vulnerabilidad social, siendo elegida por cerca de tres de cada cinco estudiantes del 60\% más pobre de la población, quienes ven en ésta la posibilidad de concretar un proceso que les permita obtener un título habilitante para un empleo. Datos del Banco Interamericano de Desarrollo (BID) ratifican que el tercio de la secundaria alta $\left(3^{\circ}\right.$ y $4^{\circ}$ año de EMTP) la eligen como una opción educativa dado que "[...] tiene un rol fundamental en la formación de la futura fuerza laboral chilena" (ARIAS et al., 2015, p. iii). Desde datos del Ministerio de Educación, las Bases curriculares del Decreto 452 del año 2013, declaran que "La Formación Diferenciada Técnico-Profesional de la Educación Media constituye un ámbito de preparación inicial para una vida de trabajo $\mathrm{y}$, más allá, un ámbito de formación integral para la vida adulta" (CHILE, 2013, p. 12), toda vez que los niveles $3^{\circ}$ y $4^{\circ}$ año de Educación Media

[...] ofrecen a los estudiantes oportunidades de realizar aprendizajes en una especialidad técnica que facilite su acceso a un primer trabajo remunerado, atendiendo a sus intereses, aptitudes y disposiciones vocacionales, y que los prepare en forma efectiva para el trabajo [...] Con igual importancia [...] considera también la continuidad de estudios técnicos como un destino posible y deseable de los egresados. (p. 14).

Sin embargo, según referencias del Sistema de Información de Educación Superior (SIES), pese a que más del 50\% de los egresados de nivel técnico profesional (TP) rinden la Prueba de Selección Universitaria al finalizar la enseñanza media, menos del 40\% continúa estudios superiores al primer o segundo año después de su egreso. En la misma línea, los resultados que alcanza la EMTP en cuanto a tasas de titulación; según datos de la División de Educación Profesional del Ministerio de Educación, muestran que el año 2016, un 24,7 \% de un total de 77.711 estudiantes que la cursaron, no llevaron a cabo sus prácticas laborales, con repercusiones para la obtención del título que lo certifica ante el mercado laboral.

Si bien el Ministerio de Educación ha declarado que un eje de la reforma educacional es la inclusión y que hoy existe un contexto propicio para cumplir con las demandas y 
valores comprometidos en ella, expresado por ejemplo, en la existencia de dispositivos políticos y programáticos educativos a nivel nacional, tales como la Ley 20.422 de inclusión e igualdad de oportunidades, la Ley 20.903 de desarrollo profesional docente y el Decreto n 170/2009 que creó el programa de Integración escolar -PIE-, entre otros, todavía es necesario avanzar en la coherencia con la práctica para que la escuela sea efectivamente un espacio en donde todos y todas aprendan con una visión universal del currículo; específicamente en lo que atañe al acceso y trayectoria a la EMTP así como su transición a la educación superior, los desafíos son amplios y variados, puesto que se encuentra en un estado de desarrollo incipiente y no se han alcanzado los estándares que se esperan; puntualmente en cuanto a inclusión, los datos de la última Encuesta Nacional de Discapacidad revelan que el sistema educativo chileno no está garantizando el acceso efectivo a matrículas y progresión de todos los estudiantes con NEE (CHILE, 2015).

Asentadas estas consideraciones sobre la EMTP y aceptando que su foco está principalmente puesto en la preparación para una meta laboral, cabe preguntarse si estas condiciones se cumplen en el caso específico de los estudiantes con NEE, particularmente de quienes presentan NEE permanentes o de discapacidad que requieren de apoyo constante y asumiendo que para ellos las exigencias de las especialidades técnicas durante la formación y las condiciones para el cumplimiento de prácticas profesionales pueden estar dificultadas, como lo están tantas otras áreas de su vida social y que en tal caso, equivaldría a suprimir o al menos mermar sus oportunidades educacionales y laborales.

\section{Método}

La perspectiva y método de la investigación realizada se basó en los principios orientadores de la teoría fundamentada, que "[...] más que una teoría se corresponde con una metodología inductiva e intensiva para descubrir teorías que dormitan en los datos" (GLASER; STRAUSS, 1967, p. 152). De acuerdo a este enfoque, el procedimiento de la G.T (Grounded Theory) permite la identificación de procesos sociales básicos, a través del Método de Comparación Constante (MCC), cuyo procedimiento codifica y refina de manera sistemática las respuestas de las entrevistas en categorías conceptuales, identificando progresivamente los temas principales desde el punto de vista del actor, descubriendo uniformidades y delimitando comprensiones teóricas emergentes de mayor abstracción (GLASER; STRAUSS, 1967), induciendo comprensiones desde los datos testimoniales de la realidad que se estudia, en este caso, las experiencias, motivaciones y expectativas de los estudiantes con NEE transitorias y permanentes, durante y después de la formación técnico profesional recibida en la Enseñanza media o secundaria.

Coherente con esta mirada, la estrategia de investigación para este estudio se definió como cualitativa, a fin de privilegiar la descripción y significado atribuido por los estudiantes a la propia acción en su proceso formativo y en su contexto natural de ocurrencia que es el sistema escolar en el que fueron entrevistados (CORBETTA, 2007).

En cuanto al tipo de estudio, éste se define como exploratorio-descriptivo, dado que en el contexto nacional no existían estudios precedentes sobre formación TP que analizaran la experiencia de estudiantes con NEE transitorias y permanentes, a la vez que 
sus resultados fueron descritos desde los atributos que los propios jóvenes les dan a estas experiencias. El diseño y trabajo de campo, se llevó a cabo entre septiembre del 2016 y febrero del 2017. La técnica fundamental escogida para el levantamiento de datos, fue la entrevista semiestructurada, en la que se dispuso de un guión flexible de preguntas acerca de las condiciones contextuales, relacionales, pedagógicas y motivacionales de su formación, permitiéndose la formulación y profundización dialógica de los temas implicados en los objetivos del estudio y otras de tipo emergente (CORBETTA, 2007). Es un procedimiento que se caracteriza por "[...] encuentros cara a cara [...]dirigidos hacia la comprensión de las perspectivas que tienen los informantes respecto de sus vidas, experiencia o situaciones, tal como las expresan en sus propias palabras"(TAYLOR; BOGDAN, 2002, p. 101).

La pauta de entrevista semiestructurada se organizó alrededor de tres grandes tópicos: 1) Motivaciones y expectativas para una formación técnico profesional; 2) Proyecciones post egreso, referidos a planes futuros y percepción de eventuales condiciones laborales dada sus necesidades especiales; y 3) Percepción sobre facilitadores y barreras del proceso formativo TP.

\section{Contexto y participantes}

El estudio se llevó a cabo en diez establecimientos de Enseñanza Media Técnico Profesional pertenecientes a comunas vulnerables en la Región Metropolitana, los que compartían similares condiciones o características tales como: a) pertenecer a Formación diferenciada Técnico Profesional; b) tener en ejecución el Programa de Integración Escolar (PIE) de directriz ministerial, asegurando así la presencia tanto de estudiantes con NEE permanentes como transitorias; c) tener dependencia municipal o particular subvencionada e índices de vulnerabilidad alta y media.

Resguardando la confidencialidad y anonimato, se entrevistó finalmente a un total de 37 estudiantes con NEE, de ambos géneros, pertenecientes a niveles de $3^{\circ}$ y $4^{\circ}$ año de Enseñanza Media, que son aquellos en los que se cursan las especialidades técnicas y que están más próximos a los procesos de práctica profesional. Se cauteló que en cada establecimiento se accediera como mínimo a dos estudiantes con NEE transitorias y dos con NEE permanentes, para triangular datos respecto de ambas condiciones y entre establecimientos. Los estudiantes entrevistados, pertenecían a las especialidades técnicas de Administración, Gastronomía, Asistencia de párvulo, Construcciones metálicas, Contabilidad, Electrónica, Electricidad, Telecomunicaciones y Vestuario.

En cuanto a las NEE que caracterizaron a esta muestra, los estudiantes con NEE de tipo permanente correspondian a Discapacidad Auditiva Severa, Discapacidad Intelectual Leve y Moderada, Síndrome de Asperger, Parálisis Braquial, Neurofibromatosis, y Trastorno motor. Mientras que los estudiantes con NEE de tipo transitorio, presentaban Funcionamiento Intelectual Limitrofe, Dificultad Especifica del aprendizaje y Trastorno de Déficit Atencional (TDA). 


\section{Resultados}

\section{Motivaciones y expectativas para una formación técnico profesional y sus proyecciones post egreso del $4^{\circ}$ año de Enseñanza Media.}

Dos aspectos resaltan respecto de las motivaciones: los agentes de influencia y los motivos para la decisión en sí, se ahondó en la elección de una formación TP en general y también por la especialidad técnica de su preferencia. Al respecto, las referencias relevan como principales agentes de influencia u orientación al entorno más inmediato del estudiante conformado por familiares directos (madre, padre, hermanos), profesores jefe o tutores de niveles previos, que mediante el consejo o el propio ejemplo orientan posibilidades; como influencia paralela también se reporta a los pares de edad, (compañeros de cursos superiores o mismo curso y amigos) "Un primo, me ayuda a estudiar, me enseña, mis papás también y toda mi familia” (Estud. NEE permanente); "Mira, a mí la construcción eléctrica no me ha gustado, es porque mi papá trabaja en esto" (Estud. NEE permanente); "También tenía amigos ahí, que me contaban y me empezó a llamar la atención y por eso que elegí estudiar la especialidad” (Estud. NEE permanente); “[...] algunos profes, mi familia no intervino mucho" (Estud. NEE transitoria).

En cuanto a los motivos para decidir por una formación TP, las respuestas se diversifican en dos nociones centrales:

a) La formación TP como vía de acceso rápido para la inserción laboral, teniendo en cuenta, por un lado, las propias capacidades, en donde se observan criterios de valoración tales como: "[...] yo quería estudiar algo para salir a trabajar, entonces fue más que todo por eso (...) es entretenida (la contabilidad), se entiende fácil, me va bien por primera vez" (Estud. NEE transitoria); "No, yo no voy a seguir estudiando no tengo memoria, pero me motiva trabajar luego, por mi mami”(Estud.NEE permanente); “[...] tener más conocimiento y así salir de $4^{\circ}$ medio y salir con un título, empezar a trabajar casi inmediatamente después de salir"(Estud NEE Transitoria). Y, por otro lado, la asequibilidad socioeconómica de la familia: "Es que lo que yo quiero estudiar es muy caro pagarlo, quiero estudiar enfermería y había que pagar mucha plata y mi mami no le daba su sueldo"(Estud. NEE permanente); "[...] o sea fue como una opción al ver a mi mamá, porque ella no pudo seguir estudiando lo que quería (...) quiero trabajar un tiempo y hacer un curso de repostería y tener mi propio negocio"(Estud. NEE transitoria); "Fue el único liceo que me aceptó, por las notas porque yo era de Iquique, me trasladé a Santiago y tenía muy malas notas”(Estud NEE transitoria). Menos recurrente como respuesta, pero también presente, aparecen algunas referencias particulares a que la especialidad o la enseñanza TP no fue una decisión propia del estudiante sino la opción más viable dadas las dificultades económicas de la familia, de rendimiento escolar o inaccesibilidad de matrículas en otros establecimientos. En estos últimos dos elementos, se entrecruza como criterio para la decisión, que el liceo contara con Programa de integración escolar (PIE), entendiendo a éste como un elemento facilitador que asegura el acceso y apoyo en la trayectoria escolar, especialmente en casos de estudiantes con NEE permanentes. Como un elemento de interés resalta que, en estos mismos casos, no siempre la especialidad técnica fue escogida, sino más bien inducida o 
"Ser alguien": motivaciones de estudiantes con Necesidades Especiales en Enseñanza secundaria técnico...

impuesta, a veces conforme a criterios de discriminación positiva y en otras por prejuicios sobre sus habilidades, ya sea desde los propios docentes o desde sus padres, que asumen que determinadas tareas no podrán ser bien resueltas o que los expone a riesgos.

b) La formación TP como una antesala para la educación superior, donde predomina la idea de obtener un título que luego permita continuar estudios superiores, ya sea en una institución técnica de nivel superior (centro de formación técnica o instituto profesional) e incluso en algunos casos, estudiar en la universidad.

En estos casos, el plan de continuar estudios superiores se proyecta como una posibilidad mediada por el anhelo de ser alguien en la vida y por los recursos económicos para asumir ese proyecto académico, el cual se obtendría bajo las estrategias de trabajar primero para ahorrar y pagar los estudios después o bien trabajar y estudiar al mismo tiempo. En varios casos, la carrera a optar no necesariamente se relaciona con lo estudiado en la especialidad TP del liceo, especialmente en aquellos a quienes la especialidad no les gustó y ven los estudios superiores como una posibilidad de desarrollar su preferencia o vocación: "Sería terminar de estudiar, hacer la técnica, una carrera aquí (...) y seguir estudiando, así me veo en un par de años”. (Estud. NEE permanente); “[...] basándome en lo que me ha dado el liceo, igual me da un poco de miedo, el salir de $4^{\circ}$ y buscar área laboral, pero quiero seguir estudiando, ser alguien” (Estud.NEE transitoria); “[...] la Gastronomía es mi pasión [...] yo quiero obtener como objetivo cuando sea grande, seguir estudiando más de esto, ser un gran chef internacional" (Estud NEE permanente).

Más allá de estos matices de connotación, las motivaciones con las que se resuelve el criterio decisional para una formación TP, se asocia con la intención final de alcanzar movilidad social para ellos mismos y sus familias, como queda ilustrado en las siguientes citas: "Ser alguien en la vida y siempre salir adelante y eso es siempre lo que me motiva" (Estud. NEE permanente); "Me motiva ser el primer profesional en mi familia, pa' sacar adelante a mi familia, después teniendo mi cartón profesional” (Estud. NEE permanente); "[...] me motiva el sacar adelante a mi familia y pienso concretarla estudiando, contabilidad ahora y luego auditoría" (Estud. NEE transitoria); "Mi familia es mi principal eje [...] también me motivan las expectativas a futuro, a nivel personal, las que son el tener una casa y ser feliz" (Estud. NEE transitoria).

\section{Proyecciones al egresar de la EMTP: Expectativas diferenciales según tipo de NEE}

En torno a este tópico, las respuestas refrendan en muchos casos las motivaciones ya antes señaladas, lo interesante es que en ciertos casos se ofrece una perspectiva más específica sobre las diferencias que asumen la concreción de estos planes en función del tipo de NEE que se presenta. Esto significa que las expectativas sobre el futuro tras egresar de la enseñanza media varían relativamente dependiendo de si la necesidad especial es de tipo permanente o transitorio. Al respecto, la gran parte de los estudiantes que presentan necesidades transitorias contempla el plan de egresar de $4^{\circ}$ año EM y continuar estudios superiores, ya sea en algo asociado con lo que actualmente están estudiando o bien estudiando algo diferente que incremente sus opciones laborales. En lo general, sus preferencias se inclinan a una formación técnica de nivel superior, aunque 
algunos también contemplan la opción de una formación universitaria, como queda refrendado en sus palabras: "Luego de dar la PSU, mis planes son seguir estudiando algo con telecomunicaciones" (Estud. NEE transitoria); "Después del liceo obviamente hacer la práctica y después estudiar lo que quiero"(Estud. NEE transitoria); "Me gustaría primero terminar mi práctica, seguir trabajando un tiempo en aquello para poder juntar plata y pagarme una carrera”(Estud. NEE Transitoria); “[...] me veo trabajando en algo que tenga que ver con esto, luego quizás seguir estudiando para especializarme más en contabilidad” (Estud. NEE transitoria).

En ellos, la idea de cumplir con la práctica laboral no aparece como una prioridad, no observan como requisito la titulación de la especialidad que cursaron. Por otra parte, en quienes sí aparece la proyección de trabajar inmediatamente después del egreso e idealmente en aquello que se está estudiado, son generalmente -aunque no exclusivamenteestudiantes que presentan NEE permanentes: "No, yo no voy a seguir estudiando no tengo memoria, pero me motiva trabajar luego, por mi mami” (Estud. NEE permanente); "Me motiva a lograr la titulación, de hacer la práctica, es difícil la electrónica” (Estud. NEE permanente); "No es muy amplio el campo laboral para una persona así pero si se le da trabajo, a veces puede llegar a ser muy difícil” (Estud. NEE permanente); "Yo creo que no son muy buenas [las condiciones], no todo está hecho para personas con discapacidades” (Estud. NEE permanente); “[...] yo creo que igual le va a costar encontrar trabajo [a una persona con discapacidad] porque la gente igual es discriminadora"(Estud. con NEE transitoria).

En este punto es importante matizar que esta visión sobre el futuro si bien es bastante general, no resulta tan optimista en todos los casos entrevistados ya que en algunos estudiantes con NEE de tipo permanente e incluso en estudiantes con NEE transitorias, se observa la incertidumbre post egreso del colegio, lo cual permitiría calificar como sombrias las expectativas en torno a las oportunidades y condiciones laborales disponibles para emplear a personas con situaciones de discapacidad, especialmente si esta condición es de tipo cognitivo o intelectual. Existe una noción temerosa o pesimista, sobre cómo las condiciones de discapacidad podrían presentar mayores dificultades para encontrar trabajo por razones de discriminación.

\section{Percepción positiva sobre el proceso formativo TP: Facilitadores y barreras}

En cuanto a la valoración de su experiencia escolar TP, los datos muestran que la mayoría de ellos percibe positivamente a sus entornos escolares; los representan como espacios protectores, en donde se sienten generalmente cómodos y seguros, en los que se valoran sus habilidades e intereses, destacando especialmente la figura de profesores significativos en su proceso, que han creído en sus capacidades y los han acompañado en los desafíos o dificultades de aprendizaje: "[...] más que adaptar, se preocupan y tienen harta disponibilidad para uno. El profe trata de hacer las clases más gratas para todos y se percata cuando algún alumno tiene una dificultad pa' aprender" (Estud. NEE permanente); “[...] gracias a los profesores he salido mucho más adelante en el aspecto matemático y otros" (Estud. NEE permanente); "Me gusta la confianza y la lealtad que hay entre 
"Ser alguien": motivaciones de estudiantes con Necesidades Especiales en Enseñanza secundaria técnico...

profesor-alumno [...] ese lazo no se pierde y eso el colegio lo fomenta mucho, lo que es el amor entre alumno-profesor" (Estud. NEE transitorio).

Los estudiantes destacan la labor de acompañamiento personalizado de los profesionales de apoyo de los PIE, a quienes consideran los facilitadores más importantes en estos procesos educativos, por su rol de mediador entre sus necesidades especiales de aprendizaje y la adecuación a exigencias escolares. Así, se destaca el componente afectivo y el ascendiente de los profesores y profesionales PIE, como una clave para construir la confianza en la solicitud de apoyo y lograr acceso a los apoyos disponibles: "De los profesores de integración son como de los únicos que puedo recibir apoyo" (Estud. NEE permanente); "La ayuda de los profesores es esencial, se organizan mucho, si ven que a un alumno le cuesta mucho, lo toman y lo ayudan más, (...) después de clases hay que quedarse a reforzamientos" (Estud. NEE permanente); "Sí, en el colegio anterior estaba en un diferencial, yo creo que más por eso quisieron seguir ayudándome aquí en el PIE” (Estud. NEE permanente); "En matemáticas y acá en taller de repente cuando me vienen a buscar para hacer otras actividades allá en la sala de atrás las tías del PIE”. (Estud. NEE permanente).

Respecto a cuán escuchados y tomados en cuenta se sienten en el espacio de la escuela, las referencias sugieren una percepción general positiva, muy condicionada por la interacción con el medio escolar, ya que depende de las situaciones de reafirmación y aceptación de los docentes, los profesionales de apoyo y los compañeros de curso. Sin embargo, es necesario señalar que existen contrastes en algunos casos de estudiantes con NEE permanentes, sobre todo en quienes presentan discapacidades de tipo cognitivo o intelectual y de comunicación, quienes perciben de manera más adversa los espacios escolares debido a experiencias y dificultades asociadas a falta de control de los estímulos ambientales (gritos o desorden en sala o patio, especialmente sensibles en el caso de estudiantes con Síndrome de Asperger) o también por falta de integración a grupos de pares, donde destacan el aislamiento y episodios de menoscabo psicológico, por lo que se sienten marginados y en situación de desventaja, como queda expresado en las siguientes citas: "[...] de primer año me han molestado, me produce rabia, ellas me molestaron, el otro día casi pelearon conmigo" (Estud. NEE permanente); “[...] a mí no me gusta que me vean así, porque estas palabras me duelen, que me digan enfermo, psicópata, idiota, por lo que yo tengo" (Estud. NEE permanente); “[...] yo encuentro que a mí siempre me han dejado a un lado [...] porque yo era rara [...] me molestaban, porque me cuestan las cosas, no me gusta que me traten diferente" (Estud. NEE permanente); "Yo venía desde la básica con problemas, parece que soy PIE... no sé, igual me molestan algunos" (Estud. NEE permanente).

\section{Bajo reconocimiento de barreras en lo escolar}

Con todo, los resultados en este sentido muestran que en general los estudiantes perciben muy pocas barreras o dificultades en el contexto escolar para su integración, lo cual está bastante mediado por el rol activo que cumplen los equipos de apoyo del PIE (Programa de integración escolar), en términos de facilitar el acceso y los procesos de aprendizaje: “[...]se me facilita el aprendizaje, desde mi punto de vista no veo ninguna 
barrera que me esté impidiendo aprender en el liceo" (Estud. NEE Transitoria); “[...] en la sala hay demasiado ruido, se ponen a gritar y eso me desconcentra de las cosas que hago" (Estud. NEE permanente); "La ayuda de los profesores es esencial, se organizan mucho, si ven que a un alumno le cuesta mucho, lo toman y lo ayudan más, (...) después de clases hay que quedarse a reforzamientos" (Estud. NEE permanente); "Sí, en el colegio anterior estaba en un diferencial, yo creo que más por eso quisieron seguir ayudándome aquí en el PIE" (Estud. NEE permanente); "En matemáticas y acá en taller de repente cuando me vienen a buscar para hacer otras actividades allá en la sala de atrás las tías del PIE” (Estud. NEE permanente).

En las escasas referencias en las que se reconocen barreras para la formación, los estudiantes las remiten más bien a sus propias dificultades de aprendizaje o bien a problemas familiares en las que se entrevé la vulnerabilidad de estos entornos, como se revela en las siguientes citas: "Es que yo venía con un diagnóstico de antes porque allá en el otro colegio [refiriéndose a la básica] igual estuve en integración”.(Estud. NEE permanente); "[...] me evaluaron en el PIE, pero igual he tenido ese problema, de que cuando estudio siempre se me olvida la materia" (Estud. NEE Transitorio); "No, si el problema era yo, si los profes de repente me prestaban mucha ayuda, siendo que cuando quedé embarazada, tenía que saltarme clases...me cuesta aprender." (Estud. NEE permanente); "[son dificultades] mis problemas en casa y en el colegio, es porque he faltado mucho, mi mamá trabajaba y no tenía con quien dejar a mi hermano y me tenía que quedar a cuidarlo" (Estud.NEE permanente).

\section{Discusión}

A nivel de conclusiones, los hallazgos permiten responder a la cuestión propuesta en este estudio, mostrando que las motivaciones para elegir una formación TP se basan principalmente en la noción de que esta educación habilita para lo laboral y es de mayor acceso dadas las condiciones de vulnerabilidad de los estudiantes y sus familias, especialmente argüido por los estudiantes en situación de discapacidad, que se plantean con más temores e incertidumbre frente al futuro. Esto contrasta con la percepción de estudiantes con NEE transitorias que, sí perciben como factible la continuidad de estudios superiores, por lo que se puede concluir que las proyecciones de estos jóvenes varían en función de sus condiciones socioeconómicas, pero sobre todo dependen de cómo auto perciben sus limitaciones y el apoyo de su entorno por el tipo de NEE que experimentan. Se detectó que lo común en sus motivaciones y expectativas, más allá del tipo de NEE y del camino que se opte al egresar de la enseñanza media, es que se perfila la educación como una estrategia condicionante para alcanzar movilidad social, lo que está notoriamente influido por los deseos personales de ser alguien y por las aspiraciones familiares, en la medida en que son significadas como un piso formativo básico asequible que le proporciona una certificación al término de estos cuatro años de enseñanza, cuestión esencial si se tiene en cuenta la extracción socioeconómica de las familias y las opciones que esto ofrece para la superación de los factores de vulnerabilidad que los rodean. Este elemento en particular encuentra coincidencia con conclusiones en 
"Ser alguien": motivaciones de estudiantes con Necesidades Especiales en Enseñanza secundaria técnico...

otros estudios como Salir del cuarto: Expectativas juveniles en el Chile de hoy (CANALES; OPAZ0; CAMPS, 2016) que muestra que jóvenes de liceos científico humanista de sectores populares, en sus discursos también articulan sus expectativas personales, familiares y las oportunidades disponibles al momento de elegir caminos tras la educación secundaria. Por ello, cobra importancia que con independencia de las NEE que un estudiante pueda o no mostrar, se deben fortalecer de modo general en la formación TP, aspectos que muestran cierta debilidad en el sistema escuela y su articulación al sistema laboral, concretamente, el desinterés o imposibilidad por insertarse a prácticas laborales y alcanzar la titulación mejorando las condiciones de empleabilidad, así como en otros casos, el de asegurar transiciones exitosas a la educación superior en aquellos estudiantes que tengan estas expectativas.

En cuanto al proceso formativo, éste es valorado en general como positivo, con reconocimiento al apoyo de los equipos de integración que operan en los colegios, su acompañamiento hace que la escuela sea apreciada como un espacio grato y facilitador, en tanto reafirma sus capacidades y los motiva a cumplir metas de estudio o trabajo; al mismo tiempo que resalta la débil percepción sobre ciertos tratamientos segregantes y diferenciales que aún se observan en los espacios escolares.

Los resultados permiten distinguir el universo de las NEE como un ámbito poco indagado en el contexto de la escolarización secundaria y de inaplazable interés si se espera avanzar hacia la superación del actual paradigma de integración en la formación $\mathrm{TP}$, el cual aún no abandona del todo las prácticas y concepciones tradicionales de la educación diferencial que lo tensionan y develan su trasfondo excluyente. Dejar de integrar para comenzar a incluir en la educación, obliga a reconsiderar todos los axiomas regentes sobre la normalidad y la uniformidad que aún se esgrimen como argumentos para resistir al cambio, tanto en niveles estructurales como en el quehacer educativo diario y concreto de los establecimientos. Si hace unos años los estudios observaban el marcado contraste entre los estudiantes de escuelas regulares o mal denominadas normales versus las escuelas especiales y su brecha se apreciaba enorme, luego los intereses se orientaron a mirar la fórmula de coexistencia entre los estudiantes con y sin NEE en un mismo espacio escolar (escuelas de integración) y a las prácticas inconscientes o simbólicas de exclusión que se mantienen entre ellos. En el mismo ámbito temático, este estudio en $\mathrm{TP}$ ha contribuido a revelar una polaridad antes inadvertida en los actuales sistemas escolares integradores, donde el criterio de oposición depende ahora del tipo de NEE que se presenta. Aunque la posibilidad de generalizar es limitada en un estudio como éste, se propone como hipótesis a profundizar el hecho de que existen diferencias de acceso, progresión y proyección entre estos estudiantes, en otras palabras, que los estudiantes que presentan NEE permanentes (sobre todo las discapacidades de tipo cognitivo, intelectual y de interacción social) se encontrarían en un estado relativo de mayor exclusión que sus pares con NEE transitorias y están enfrentando dificultades que no están siendo resueltas por el contexto escolar, pese a la existencia de dispositivos especiales, como el PIE. Sin pretender negar el aporte de los equipos PIE en cuanto a instalar la idea de inclusión y en cómo ellos pueden ser un puente en la transición paradigmática dada su vocación y experiencia, es inevitable plantearse la cuestión paradojal que se genera a 
nivel sistémico en cuanto a que el PIE puede ser leído como una solución problemática o una componente contra ideológica para la meta de inclusión que se busca promover, dado que al mismo tiempo que es un dispositivo valorado por facilitar el acceso y aprendizaje de los estudiantes, su sola existencia instituye la oposición normalidad/anormalidad que pretende dejar atrás. Los relatos revisados aportan evidencia que refrenda que pese a la existencia y esfuerzo de los PIE en los establecimientos TP, la inclusión de los estudiantes en situaciones de discapacidad constituye el desafío más difícil en cuanto a asegurar acceso y apoyo efectivo. Los datos de esta investigación, por ejemplo, son consistentes cuando evidencian que en los sistemas escolares no están representados todos los tipos de discapacidades y las experiencias de los que sí acceden no siempre resultan ser óptimas para su desarrollo, tanto en el proceso formativo como en miras a alcanzar empleabilidad $\mathrm{y}$ vida independiente, respondiendo a sus propias expectativas y metas personales.

Todo lo anterior lleva inevitablemente a la cuestión de cuál debería ser entonces la estrategia adecuada para el logro de una educación inclusiva y de calidad en el caso de estudiantes con NEE. En estudios precedentes, se reconoce que la clave está en desarrollar un sistema escolar pensado con un diseño más universal y equitativo, donde las diferencias no sean asumidas como anormalidades que deben ser corregidas o asimiladas, la respuesta sería "Poner el énfasis en los modos de superar tanto los procesos y las situaciones de abierta exclusión, segregación y marginación educativa" (CASANOVA; CABRA DE LUNA, 2009, p. 46). En otras reflexiones disciplinarias, se subraya que la inclusión educativa

[...] trata de algo distinto y que quiere ir mucho más allá de lo conseguido por los procesos de integración escolar [...] los que mayoritariamente (han sido tratados), como un asunto técnico relacionado con la tarea de conseguir que unos pocos alumnos que estaban fuera de los centros ordinarios o regulares del sistema, se preparasen para estar dentro, sobre todo algunos -no todosde los considerados con necesidades educativas especiales[...] se persigue algo cualitativamente distinto a lo visto hasta ahora porque estos procesos de integración débil se han realizado bajo la perspectiva de que este nuevo alumnado se "asimile o acomode" a los patrones culturales de la normalidad imperante.” (ECHEITA, 2013, p. 103).

Al profundizar sobre las expectativas de los estudiantes cuando proyectan la vida laboral, preocupa la creencia de que los empleadores tienen prejuicios sobre las capacidades de desempeño y adecuación de alguien que tenga alguna situación especial, dificultando así la inserción laboral, aun cuando algunos relatos matizan con la idea de que ha habido leves cambios a favor de la incorporación laboral de personas con discapacidad y de que no debería haber discriminación, es evidente la incertidumbre que se produce en torno a ciertas discapacidades más severas, especialmente en el ítem de las condiciones del empleo. Teniendo en cuenta esta realidad, se hace necesario contemplar el fortalecimiento de programas de inclusión que enfaticen un entrenamiento específico en vida independiente y habilidades sociales. En este sentido, un estudio citado por Luftig y Muthert (2005) en Estados Unidos mostró que en cuanto a empleabilidad, salario y vida independiente las personas que se han graduado en ambientes inclusivos de tipo vocacional y tecnológico en la secundaria, lo han hecho bastante bien en comparación a encuestados formados en 
"Ser alguien": motivaciones de estudiantes con Necesidades Especiales en Enseñanza secundaria técnico...

otros contextos no inclusivos. "Lo que es esperanzador es que tal enfoque y entrenamiento fue realizado en un escenario inclusivo con otros pares no discapacitados que también estaban recibiendo entrenamiento vocacional sólido” (LINDSTROM; BENZ, 2002, p. 78) lo que refuerza la necesidad de avanzar en este mismo sentido en los contextos escolares técnico-profesionales chilenos.

A modo de reflexión final, quisiéramos señalar que el influjo del mundo moderno ha transformado la concepción histórica de una educación concebida como factor de desarrollo humano en sí mismo a un concepto de educación de carácter más instrumental; desde esa perspectiva, la educación para el trabajo, constituye hoy en día más que un derecho una obligación social, dado que se entiende que los estamentos o sistemas de una sociedad deben organizarse para garantizar las oportunidades de acceso en los diferentes niveles formativos para que todos los individuos puedan lograr mayores y mejores oportunidades de inserción laboral, lo que en definitiva se traduce en oportunidades de movilidad social y aporta hacia mejoras proporcionales en la calidad de vida.

En Chile, si hablamos de las NEE en el sistema escolar general o en la EMTP en particular, se debe reconocer que los esfuerzos políticos ministeriales, han demostrado una vocación e interés adaptativo creciente, pero pese a ello aún estamos en proceso de reflexionar y comprender cómo se superan las lógicas excluyentes de la educación diferencial. En este sentido, se debe admitir que la integración, aunque débil e incompleta, es en parte un avance o paso respecto a sistemas segregados entre la educación especial y la educación regular, pero que si no se evoluciona sobre sus condiciones y necesidades ni se toma en cuenta la perspectiva de quienes lo viven ni tampoco existe voluntad política para el cambio, esta relativa integración no necesariamente se constituirá en la antesala de un sistema inclusivo ya que ese salto no resulta automático ni espontáneo. Por lo demás, es el momento adecuado para señalar que la diversidad de necesidades o condiciones que los estudiantes presentan en los sistemas escolares, no solo se refieren a condiciones motoras o físicas, cognitivas, sensoriales, etc., sino que a otra serie de características humanas (diferencias socioeconómicas, diferentes orígenes étnicos, diversas identidades de género y orientación sexual, por nombrar algunas) que de manera creciente irán diversificando las aulas de nuestras escuelas y demandarán una respuesta diferente a la que hoy se está dando. Por esto, las actuales nociones de integración en lo escolar ya se han visto interpeladas y exigidas de mutar hacia un nuevo paradigma o concepción, que logre efectivamente dar respuesta a las demandas de una sociedad cada vez más heterogénea. En este contexto, este estudio permitió considerar la perspectiva de los estudiantes con NEE y entender cómo comprenden y vivencian su sistema escuela y que en tanto sistema requiere de insumos, retroalimentación y aprendizaje constantes. En este sentido, se espera seguir aportando en la generación de diálogos en torno a las inquietudes y cuestionamientos sobre las necesidades y prácticas escolares actuales empujando mejoras que transiten desde un eje integrativo en la educación hacia un horizonte más inclusivo. 


\section{Referencias}

ARIAS, Elena et al. Educación técnica profesional en Chile. Santiago de Chile: Banco Interamericano de Desarrollo, 2015.

CANALES, Manuel; OPAZO, Antonio; CAMPS, Juan. Salir del cuarto: expectativas juveniles en el Chile de hoy. Última Década, Santiago de Chile, n. 44, p. 73-106, 2016.

CASANOVA, María Antonia; CABRA DE LUNA, Miguel Ángel. Educación y personas con discapacidad: presente y futuro. Madrid: Fundación Once, 2009.

CHILE. Ministerio de Desarrollo Social. Estudio nacional de la discapacidad (Endisc). Santiago de Chile: Gobierno de Chile, 2015.

CHILE. Ministerio de Educación. Decreto 170. Santiago de Chile: Biblioteca del Congreso, 2009. Disponible en: <http://www.leychile.cl/Navegar?idNorma=1012570 >. Acceso en: 8 sept. 2016.

CHILE. Ministerio de Educación. Orientaciones Técnicas para Programas de Integración Escolar (PIE). Santiago de Chile: Gobierno de Chile, 2013.

CORBETTA, Piergiorgio. Metodología y técnicas de Investigación social. Madrid: Mc Graw Hill, 2007.

ECHEITA, Gerardo. Inclusión y exclusión educativa. De nuevo "voz y quebranto". Reice, Madrid, v. 11, 2013. Disponible en: <http://www.redalyc.org/articulo.oa?id=55127024005>. Acceso en: 9 mzo. 2018.

GLASER, Barney; STRAUSS, Anselm. The discovery of grounded theory: strategies for qualitative research. Aldine: Observations, 1967. (Chicago; III).

GÓNGORA, Juana. Necesidades educativas especiales. Santiago de Chile: [s. n.], 2009. Disponible en: < https:// es.scribd.com/presentation/70563749/necesidades-educativas-especiales>. Acceso en: 28 jun. 2016.

LARRAÑAGA, Osvaldo; CABEZAS, Gustavo; DUSAILLANT, Francisca. Informe completo del estudio de la educación técnico profesional: Santiago de Chile: PNUD, 2013. Programa Nacional de las Naciones Unidas para el Desarrollo (PNDU). Área de reducción de la pobreza y la desigualdad.

LINDSTROM, Lauren E.; BENZ, Michael R. Phases of career development: case studies of young women with learning disabilities. Exceptional Children, Eugene, v. 69, n. 1, p. 67-83, 2002. Disponible en: <https:// www.researchgate.net/publication/295304535_Phases_of_career_development_Case_studies_of_ young_women_with_learning_disabilities>. Acceso en: 23 febr. 2016.

LUFTIG, Richard; MUTHERT, Dorothy. Patterns of employment and independent living of adult graduates with learning disabilities and mental retardation of an inclusionary high school vocational program. Research in Developmental Disabilities, v. 26, n. 4, p. 317-325, 2005.

MORI, Allen. Career education for the learning disabled-where are we now. Learning Disability Quarterly, Austin, v. 3, n. 1, p. 91-101. Disponible en: <http://www.jstor.org/stable/1510430>. Acceso en: 18 my. 2018. 
OCDE. Organización de las Naciones Unidas para la Educación, la Ciencia y la Tecnología. No more failures: ten steps to equity in education. Paris: OCDE, 2007. Disponible en: <https://www.oecd.org/ education/school/45179151.pdf>. Acceso en: 8 my. 2019.

TAYLOR, Steven J; BOGDAN, Robert. Introducción a los métodos cualitativos de investigación. Barcelona: Paidós, 2002.

UNESCO. La educación inclusiva: el camino hacia el futuro. Ginebra: Unesco, 2008. Conclusiones finales de la 48. Conferencia Internacional. Disponible en: <http://www.ibe.unesco.org/>. Acceso en: 8 my. 2019.

WAGNER, Mary; NEWMAN, Lynn; JAVITZ, Harold. The benefits of high school Career and Technical Education (CTE) for youth with learning disabilities. Journal of Learning Disabilities, Austin, v. 49, n. 6, p. 1-13, 2015.

Recibido en: 09.03.2018 Revisiones en: 01.10.2018 Aprobado en: 06.11.2018

Marcela Salazar Ramos es antropóloga social de la Universidad Austral de Chile (UACH) y máster en Docencia para la Educación Superior de la Universidad Andrés Bello (UNAB). Doctorante de Educación UAH-UDP. Académica del área de Humanidades y Educación en la Universidad Tecnológica de Chile-Inacap e investigadora en temáticas de Educación, Diversidad e Inclusión. Autora del libro Desafíos para la Inclusión educativa y laboral en Chile.

Pedro Quiroga Menéndez es sociólogo de la Pontificia Universidad Católica de Chile. Máster en Políticas Educativas en la Universidad Alberto Hurtado y máster en Sociología e Investigación de la London School of Economics and Political Science (LSE). Académico y consultor para proyectos sociales y educacionales con impacto nacional.

Ximena Risco Neira es trabajadora Social de la U. de Valparaíso, Master en planificación y gestión educacional de la U.Diego Portales y Doctora en Ciencias de la Educación de la U. de Aconcagua. Académica con trayectoria y liderazgo de proyectos educativos centrados en currículo, calidad y estándares en Educación superior. 